\author{
Marquette University \\ e-Publications@Marquette
}

College of Nursing Faculty Research and

Publications

Nursing, College of

$12-2019$

\title{
Effects of Women's Weight Changes on Adverse Outcomes in a Second Pregnancy
}

Marianne E. Weiss

Marquette University, marianne.weiss@marquette.edu

Olga Yakusheva

Marquette University, olga.yakusheva@marquette.edu

Kandice A. Kapinos

RAND Corporation

Follow this and additional works at: https://epublications.marquette.edu/nursing_fac

Part of the Nursing Commons

\section{Recommended Citation}

Weiss, Marianne E.; Yakusheva, Olga; and Kapinos, Kandice A., "Effects of Women's Weight Changes on Adverse Outcomes in a Second Pregnancy" (2019). College of Nursing Faculty Research and Publications. 627.

https://epublications.marquette.edu/nursing_fac/627 
Marquette University

e-Publications@Marquette

\section{Nursing Faculty Research and Publications/College of Nursing}

This paper is NOT THE PUBLISHED VERSION; but the author's final, peer-reviewed manuscript. The published version may be accessed by following the link in th citation below.

Journal of Obstetric, Gynecologic and Neonatal Nursing, Vol. 48, No. 6 (November 2019): 615-626. DOI. This article is (C) Elsevier and permission has been granted for this version to appear in ePublications@Marquette. Elsevier does not grant permission for this article to be further copied/distributed or hosted elsewhere without the express permission from Elsevier.

\section{Effects of Women's Weight Changes on Adverse Outcomes in a Second Pregnancy}

Marianne Weiss

College of Nursing, Marquette University, Milwaukee WI

Olga Yakusheva

School of Nursing and School of Public Health, University of Michigan, Ann Arbor, MI

Kandice Kapinos

RAND Corporation, Arlington, VA

\section{Abstract \\ Objective}

To estimate the effects of women's weight changes in four sequential perinatal periods across first and second pregnancies (pregravid, first gestation, interpregnancy, second gestation) on adverse maternal and neonatal outcomes in the second pregnancy while accounting for interdependencies in weight across the four periods (Aim 1) and to test the influence of the sequential path of weight changes through the four perinatal periods of risk on maternal and neonatal outcomes in the second pregnancy (Aim 2).

Design

Secondary data analysis. 


\section{Setting}

Thirty-one Wisconsin hospitals.

\section{Sample}

Women with 24,795 linked records from first and second births from 2006 through 2013.

\section{Methods}

We used a fully recursive system of linear and logistic regression equations to examine the relationships among weight changes in the four perinatal periods with maternal (gestational diabetes mellitus, gestational hypertension, cesarean birth) and neonatal (macrosomia, small for gestational age, large for gestational age, low birth weight, congenital anomalies, and perinatal death) adverse outcomes in the second pregnancy.

\section{Results}

Pregravid weight was weakly and inconsistently associated with weight changes in subsequent periods. Each 5$\mathrm{kg}$ incremental weight change in the first pregnancy, interpregnancy, and second pregnancy contributed to a 0.75 - to $5-\mathrm{kg}$ weight change in subsequent periods, $9 \%$ to $25 \%$ change in risk for adverse maternal outcomes, and $8 \%$ to $47 \%$ change in risk for adverse neonatal outcomes in the second pregnancy. Fluctuations in weight across pregnancies and associations with outcomes were strongest among normal-weight and overweight women.

\section{Conclusion}

Weight changes across two pregnancies affected maternal and neonatal outcomes in the second pregnancy in all body mass index categories; the larger weight fluctuations observed in normal and overweight women were associated with greater risk of adverse outcomes. Attention to pregnancy weight during and between pregnancies is important for targeted weight counseling to reduce risks in subsequent pregnancies.

\section{Keywords}

gestational weight gain, interpregnancy weight retention, maternal outcomes, neonatal outcomes, perinatal, pregravid weight

Research and practice guidelines about women's weight have largely focused on pre-pregnancy body mass index (BMI) and gestational weight gain (GWG) as risks and mediating factors for birth outcomes (American College of Obstetricians and Gynecologists, 2013). The effect of women's cycling up and down in weight across multiple pregnancies has received little attention, yet evidence about the role of weight fluctuation could inform clinical practice and contribute to more refined risk prediction and weight management approaches to improve maternal and infant outcomes of multiparous women.

The concept of perinatal periods of risk (CityMatCH, n.d.) is a useful framework through which to study changes in women's weight associated with childbearing. For women with two pregnancies, four periods of weightrelated risk exist during the perinatal cycle: pregravid, first gestation, interpregnancy, and second gestation. Weight changes in each period have the potential for independent and sequential effects on the health of the woman and her newborn, yet the full sequential relationship of each of these interdependent maternal weight changes across two pregnancies has not yet been examined.

Women's weight changes before pregnancy and during the first gestation, interpregnancy, and the second gestation can introduce risks for adverse second pregnancy outcomes.

Relationships between weight changes across the four perinatal risk periods are complex and interdependent. More than $50 \%$ of women in the United States are overweight or obese before pregnancy. Although overweight 
and obese women gain less gestational weight than normal-weight women, they are more likely to exceed clinical recommendations for GWG (Gunderson, 2009, Rasmussen and Yaktine, 2009). Forty-eight percent of U.S. women exceed pregnancy weight gain recommendations (Centers for Disease Control and Prevention, n.d.), and excessive GWG in one pregnancy was linked to increased risk of excessive GWG in subsequent pregnancies (Chin et al., 2010). High pregravid BMI and excessive GWG have independent associations and jointly exacerbate increased weight retention after birth (Endres et al., 2015, Gunderson, 2009, Martin et al., 2014, Nehring et al., 2011, Siega-Riz et al., 2009).

Studies on the associations between weight changes and maternal and neonatal outcomes have focused on women's weight during only one or two of the perinatal periods of risk, and most researchers examined samepregnancy outcomes. For example, pregravid obesity was associated with elevated risk of gestational diabetes mellitus, gestational hypertension, preeclampsia, and cesarean birth (Stubert et al., 2018, Tabet et al., 2015); fetal, neonatal, and infant deaths (Chu et al., 2007; Johansson et al., 2014, Stubert et al., 2018, Tabet et al., 2015); birth weight, macrosomia, and large-for-gestational-age (LGA) infants (Monte et al., 2011, Rowlands et al., 2010); hypoglycemia (Collins, Oehmen, \& Mehta, 2018); and neural tube, heart, and other structural anomalies (Correa and Marcinkevage, 2013, Stothard et al., 2009). However, studies of the effects of pregravid weight have frequently not accounted for the correlation between pregravid BMI and GWG, even though excessive GWG was also independently linked to greater incidence of preeclampsia, cesarean birth, and LGA infants (McDowell, Cain, \& Brumley, 2019). In three population-based studies of subsequent pregnancy outcomes, interpregnancy weight increase from the beginning of the first pregnancy to the beginning of the next pregnancy of 2 to $3 \mathrm{BMI}$ points was linked to a two- to threefold increase in the odds of gestational diabetes, gestational hypertension, and cesarean birth (Bogaerts et al., 2013, Villamor and Cnattingius, 2006, Wallace et al., 2016), newborn macrosomia (Bogaerts et al., 2013, Wallace et al., 2016), LGA newborns, and stillbirth (Cnattingius and Villamor, 2016, Villamor and Cnattingius, 2006, Wallace et al., 2016). Notably, the relationship between interpregnancy weight increase and maternal and neonatal outcomes was evident in underweight and normal-weight women. However, in each of these studies, the researchers did not report separate effects of each individual weight period across the two pregnancies.

Longitudinal studies that incorporate weight data from all perinatal periods are needed to better understand the effect of the full weight trajectory on subsequent health outcomes of women and neonates. The purpose of this study was to estimate the effects of women's weight changes in four sequential perinatal periods across first and second pregnancies (pregravid, first gestation, interpregnancy, second gestation) on adverse maternal and neonatal outcomes in the second pregnancy while accounting for interdependencies in weight across the four periods. The specific aims of the analysis were to estimate the sequential path of women's weight changes through the four perinatal periods of risk across two pregnancies (Aim 1) and to test the influence of the sequential path of weight changes through the four perinatal periods of risk on maternal and neonatal outcomes in the second pregnancy (Aim 2; see Figure 1).

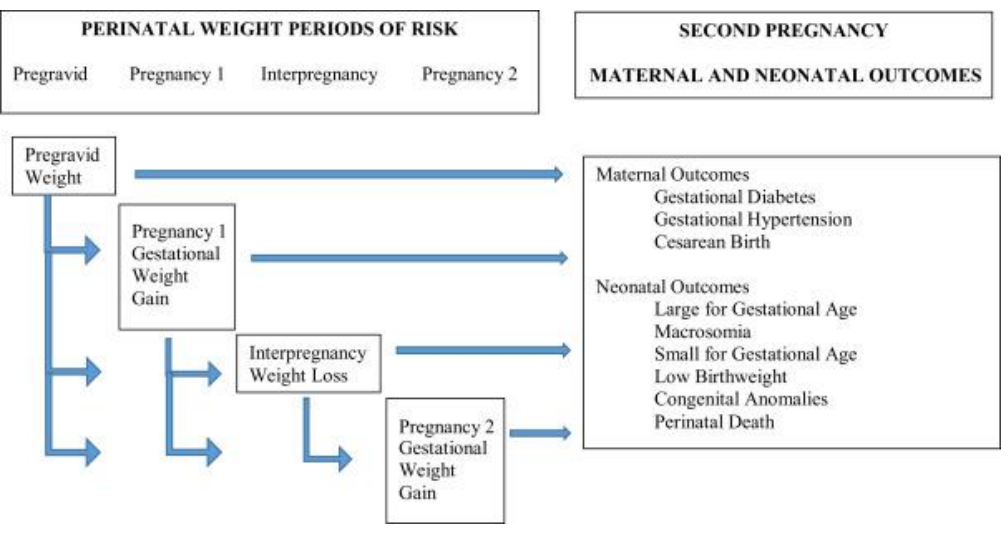


Figure 1. Model of the direct and sequential effects of perinatal weight periods of risk on maternal and neonatal outcomes in the second pregnancy.

\section{Methods}

\section{Design and Setting}

We used data from PeriData.net, a Web-based data platform developed by the Wisconsin Association for Perinatal Care, which contained data from 87 of 100 birth hospitals (92\% of total births) in Wisconsin from 2006 through 2013. At the time of data extraction in March 2013, the PeriData.net system included 414,017 birth records. Thirty-one hospitals agreed to provide limited data sets to create the study database, which included 236,820 (57\%) birth records. Approval was obtained from the Marquette University institutional review board (IRB); IRB determinations were obtained from participating hospitals or deferred to the primary IRB.

\section{Sample}

We linked births for the same woman that occurred in any of the hospitals included in the data set. The analysis was restricted to women for whom data were available for the first and second birth and in which each pregnancy resulted in a singleton birth between 20 and 42 weeks gestation ( $N=29,090$ women). See the Supplemental Appendix for a detailed description of sample derivation. After excluding observations with missing data, our final sample consisted of 24,795 women. Multiple imputation of missing values produced nearly identical results, which are not reported here.

\section{Measures}

\section{Outcome measures}

We created the following dichotomous maternal and neonatal health measures: gestational diabetes; gestational hypertension; cesarean birth; LGA and small for gestational age (SGA), calculated according to Talge, Mudd, Sikorskii, and Basso (2014); macrosomia (birth weight $>4,000 \mathrm{~g}$ ); low birth weight (<2,500 g); the presence of any congenital anomalies; and perinatal death defined as stillborn or neonatal death before the time of maternal discharge after birth.

\section{Weight measures}

We constructed four measures of weight changes in kilograms: pregravid weight for the first pregnancy, GWG during the first pregnancy (P1 GWG), interpregnancy weight loss, and GWG during second pregnancy (P2 GWG). Pregravid weight was the woman's weight before pregnancy as recorded in PeriData.net by the birth hospitals. This information was derived from prenatal records submitted by maternity care providers in preparation for birth. Pregravid weight was self-reported to the maternity care provider or measured at the first prenatal visit. P1 GWG and P2 GWG were calculated as weight at birth minus pregravid weight for the same pregnancy. Weight at birth was measured on admission in labor or recorded from the weight at the last prenatal visit, depending on the practice at the birth hospital. Interpregnancy weight loss was calculated as the woman's weight at birth of P1 minus the woman's pregravid weight for P2.

\section{Control variables}

We controlled for gestational age at birth in P1 and P2 and the interpregnancy interval (months from P1 birth to P2 birth, minus gestational age in P2). Additionally, we used the following maternal demographic characteristics from P2 data as controls: age, marital status (yes/no), race (White, Black, Asian, American Indian/Native America, Hawaiian/Pacific Islander, and other), Hispanic ethnicity (yes/no), participation in the Special Supplemental Nutrition Program for Women, Infants, and Children (yes/no), type of health insurance (private, Medicaid, other public, or uninsured/self-pay), education level (less than high school, completed high school, 
partial college, completed 4-year college, graduate degree), use of tobacco during pregnancy (yes/no), use of alcohol during pregnancy (yes/no), and height in inches.

\section{Analysis}

We conducted all analyses with the use of Stata Version 14 (StataCorp, 2015). We calculated summary statistics for the outcome measures and weight in each perinatal period and presented them in tabular form (see Table 1) for all women in the sample and by pregravid BMI category (P1) using guidelines from the National Heart, Lung, and Blood Institute (1998). The underweight category was a small proportion of the total sample $(n=1,154$, 4.65\%); therefore, we included underweight in the normal-weight category (BMI $\left.<25 \mathrm{~kg} / \mathrm{m}^{2}\right)$ for the analyses. Maternal demographic controls are presented in the Supplemental Appendix. 
Table 1. Sample Characteristics by Pregravid BMI Categories

\begin{tabular}{|c|c|c|c|c|}
\hline & All Women $(N=24,795)$ & Pregravid BMI & & \\
\hline & & $\begin{array}{l}\text { Underweight/Normal } \\
\text { Weight: } \mathrm{BMI}< \\
25.0 \mathrm{~kg} / \mathrm{m}^{2}(n=14,566)\end{array}$ & $\begin{array}{l}\text { Overweight: } \mathrm{BMI}= \\
25.0-29.9 \mathrm{~kg} / \mathrm{m}^{2}(n= \\
5,866)\end{array}$ & $\begin{array}{l}\text { Obese: } \mathrm{BMI} \geq \\
30.0 \mathrm{~kg} / \mathrm{m}^{2}(n= \\
4,363)\end{array}$ \\
\hline \multicolumn{5}{|l|}{ Weight in kg, mean (SD) } \\
\hline Pregravid weight & $68.53(16.83)$ & $58.46(7.35)$ & $73.41(7.48)$ & $95.58(15.88)$ \\
\hline Gestational weight gain in Pregnancy 1 & $15.35(6.76)$ & $15.93(6.01)$ & $15.95(6.90)$ & $12.60(8.13)$ \\
\hline Interpregnancy weight loss & $12.26(9.05)$ & $12.70(8.16)$ & $12.56(8.97)$ & $10.41(11.44)$ \\
\hline Pre-Pregnancy 2 weight & $71.62(18.32)$ & $61.69(9.96)$ & $76.80(11.64)$ & $97.77(18.60)$ \\
\hline $\begin{array}{l}\text { Pregravid weight change (pre-Pregnancy } 2 \\
\text { weight - Pregnancy } 1 \text { weight) }\end{array}$ & $3.09(8.52)$ & $3.23(7.36)$ & $3.39(8.81)$ & $2.20(11.26)$ \\
\hline Gestational Weight Gain in Pregnancy 2 & $13.17(6.63)$ & $13.78(5.81)$ & $13.32(7.02)$ & $10.95(8.05)$ \\
\hline Height in $\mathrm{cm}$, mean (SD) & $164.39(7.56)$ & $164.48(7.74)$ & $164.36(7.24)$ & $164.13(7.35)$ \\
\hline $\begin{array}{l}\text { Gestational age in Pregnancy } 1 \text { in weeks, mean } \\
\text { (SD) }\end{array}$ & $38.96(2.20)$ & $38.98(2.10)$ & $39.05(2.07)$ & $38.77(2.64)$ \\
\hline Interpregnancy interval in months, mean (SD) & $20.61(13.20)$ & $20.76(13.15)$ & $20.57(13.14)$ & $20.17(13.16)$ \\
\hline $\begin{array}{l}\text { Gestational age in Pregnancy } 2 \text { in weeks, mean } \\
\text { (SD) }\end{array}$ & $38.85(1.73)$ & $38.83(1.81)$ & $38.94(1.63)$ & $38.70(2.04)$ \\
\hline \multicolumn{5}{|l|}{$\begin{array}{l}\text { Maternal/neonatal outcomes in Pregnancy } \\
1, n(\%)\end{array}$} \\
\hline Gestational diabetes & $778(314)$ & $281(1.93)$ & 199 (3.39) & $298(6.83)$ \\
\hline Gestational hypertension & $1,829(7.38)$ & $748(5.14)$ & $492(8.39)$ & $589(13.50)$ \\
\hline Cesarean & $5,423(21.87)$ & $2,488(17.08)$ & $1,446(24.65)$ & $1,489(34.13)$ \\
\hline Any congenital anomalies & $718(2.90)$ & $398(2.73)$ & $174(2.97)$ & $146(3.35)$ \\
\hline Perinatal death & $199(0.80)$ & $99(0.68)$ & $40(0.68)$ & $60(1.38)$ \\
\hline Large for gestational age & $2,171(8.76)$ & $990(6.80)$ & $636(10.84)$ & 545 (12.49) \\
\hline Macrosomia & $2,013(8.12)$ & $928(6.37)$ & $581(9.90)$ & $504(11.55)$ \\
\hline Small for gestational age & $2,669(10.76)$ & $1,692(11.62)$ & $542(9.24)$ & 435 (9.97) \\
\hline Low birth weight & $1,679(6.77)$ & $1,003(6.89)$ & $341(5.81)$ & $335(7.68)$ \\
\hline \multicolumn{5}{|l|}{$\begin{array}{l}\text { Maternal/neonatal outcomes in Pregnancy } \\
2, n(\%)\end{array}$} \\
\hline Gestational diabetes & $1,170(4.72)$ & $452(3.10)$ & $306(5.22)$ & $412(9.44)$ \\
\hline Gestational hypertension & $1,128(4.55)$ & $451(3.10)$ & $305(5.22)$ & $372(8.53)$ \\
\hline Cesarean & $5,936(23.94)$ & 2,719 (18.67) & $1,560(26.59)$ & 1,657 (37.98) \\
\hline
\end{tabular}




\begin{tabular}{|l|l|l|l|l|}
\hline Any congenital anomalies & $667(2.69)$ & $398(2.73)$ & $147(2.51)$ & $122(2.80)$ \\
\hline Perinatal death & $113(0.46)$ & $53(0.36)$ & $22(0.38)$ & $38(0.87)$ \\
\hline Large for gestational age & $3,194(12.88)$ & $1,512(10.38)$ & $894(15.24)$ & $788(18.06)$ \\
\hline Macrosomia & $2,694(10.87)$ & $1,298(8.91)$ & $759(12.94)$ & $637(14.60)$ \\
\hline Small for gestational age & $1,808(7.29)$ & $1,186(8.14)$ & $362(6.17)$ & $260(5.96)$ \\
\hline Low birth weight & $1,144(4.61)$ & $723(4.96)$ & $227(3.87)$ & $194(4.45)$ \\
\hline
\end{tabular}

Note. From Wisconsin Births in the PeriData.net Database, 2006 through 2013. BMI = body mass index; $S D=$ standard deviation. 
We jointly tested the sequential path of maternal weight changes through the perinatal periods (Aim 1) and the influence on P2 outcomes (Aim 2) using a fully recursive simultaneous equations system. This analytic method allowed us to examine the direct and indirect relationships among the weight variables and the outcomes in a system of equations representing these interrelated sequential paths of influence. (Clayton \& Pett, 2011). For example, pregravid weight affects P1 outcomes (e.g., cesarean birth) directly and indirectly through P1 GWG, and both of these paths contribute to P2 GWG and sequentially to P2 outcomes. The full conceptual model for the analysis is presented in Figure 1, and the full analytic model with all variables is included in the Supplemental Appendix in Figure A1.

We used linear and logistic regression models for continuous (weight) and binary (outcomes) variables, respectively. Regression coefficients (for weight measures) represent absolute effect sizes measured in kilograms for ease of interpretation. Odds ratios are reported for occurrences of maternal and neonatal outcomes. All models included the full set of control variables.

We selected maternal characteristics for inclusion with the use of a backward stepwise elimination process using Stata's stepwise backward selection command and 0.05 significance (Afifi et al., 2012, Hosmer et al., 2013, Lindsey and Sheather, 2010). We excluded those that were not individually statistically significant and did not improve the model $F$ statistic in any of the equations from the model.

We estimated the full simultaneous equations model using the seemingly unrelated estimation technique ("suest" command) with robust standard errors that accounted for hospital-level clustering and the interrelated nature of the error terms among the equations (Weesie, 1999). We used hospital-level fixed effects to account for hospital-specific contextual factors. We conducted all tests using standard hypothesis testing techniques and a variance-covariance matrix of regression coefficients that included within- and between-equations parameter estimates, thus adjusting for multiple hypothesis testing (Clogg, Petkova, \& Haritou, 1995).

Normal-weight and overweight women had greater weight fluctuations than obese women, and these fluctuations were associated with greater risk of adverse perinatal outcomes in a second pregnancy.

\section{Results}

The descriptive statistics of the outcome measures and weight changes during the perinatal periods for our sample are shown in Table 1. (Maternal demographic characteristics at P2 are presented in the Supplemental Appendix). The average pregravid weight was $68.53 \mathrm{~kg}$ (standard deviation $=16.83$ ). BMI category means were $58.46,73.41$, and $95.58 \mathrm{~kg}$ for normal-weight $\left(\mathrm{BMI}<25.0 \mathrm{~kg} / \mathrm{m}^{2}\right)$, overweight $\left(\mathrm{BMI}=25.0-29.9 \mathrm{~kg} / \mathrm{m}^{2}\right)$, and obese (BMI $\geq 30.0 \mathrm{~kg} / \mathrm{m}^{2}$ ) women, respectively. The average GWG for the total sample was $15.35 \mathrm{~kg}$ in first pregnancies and $13.17 \mathrm{~kg}$ in second pregnancies. Interpregnancy weight loss was $12.26 \mathrm{~kg}$ after a mean interpregnancy interval of 20.5 months (standard deviation = 13.4), which led to an average increase of $3.09 \mathrm{~kg}$ from pregravid weight to pre-P2 weight. Normal/underweight and overweight women had larger weight fluctuations than obese women in all perinatal periods: they gained approximately $3 \mathrm{~kg}$ more during both gestations and lost approximately $2 \mathrm{~kg}$ more during the interpregnancy period. Rates of gestational diabetes, gestational hypertension, cesarean birth, perinatal death, LGA, and macrosomia increased as maternal pregravid $\mathrm{BMI}$ increased. Incidence of SGA decreased with higher maternal BMI.

The sequential effect of women's weight in each perinatal period on subsequent periods (Aim 1) is shown in Table 2. The results are reported as the effect, measured in kilograms, of a 5-kg difference in weight in one period on the next period. For example, for each $5 \mathrm{~kg}$ (11 pounds) of greater pregravid weight, the average P1 GWG in the sample was $0.37 \mathrm{~kg}$ smaller. Pregravid weight was less strongly associated with subsequent weight changes (P1 GWG) than weight effects across the other periods (see Supplemental Appendix). Maternal weight changes during $\mathrm{P} 1$, the interpregnancy period, and $\mathrm{P} 2$ were strongly and positively interrelated: for each $5-\mathrm{kg}$ 
increase in P1 GWG, interpregnancy weight loss increased by $2.94 \mathrm{~kg}$, and for each $5-\mathrm{kg}$ increase in interpregnancy weight loss, P2 GWG increased by $1.87 \mathrm{~kg}$.

Table 2. Trajectory of Effects of Perinatal Weight Change on Weight in Subsequent Perinatal Periods

\begin{tabular}{|l|l|l|l|}
\hline Perinatal Period & Effect On & & \\
\hline & Pregnancy 1 GWG & $\begin{array}{l}\text { Interpregnancy Weight } \\
\text { Loss }\end{array}$ & Pregnancy 2 GWG \\
\hline All women $(\boldsymbol{N}=\mathbf{2 4 , 7 9 5 )}$ & & & \\
\hline Pregravid & $-0.370^{*}[-0.400,-0.339]$ & & \\
\hline Pregnancy 1 & & $2.937^{*}[2.816,3.058]$ & \\
\hline Interpregnancy & & & $1.867^{*}[1.767,1.967]$ \\
\hline $\begin{array}{l}\text { Normal weight (includes } \\
\text { underweight; } \boldsymbol{n}=\mathbf{1 4 , 5 6 6 )}\end{array}$ & & & \\
\hline Pregravid & $0.391^{*}[0.286,0.497]$ & $3.184^{*}[3.006,3.362]$ & \\
\hline Pregnancy 1 & & & $1.730^{*}[1.588,1.872]$ \\
\hline Interpregnancy & & & \\
\hline Overweight $(\boldsymbol{n}=\mathbf{5 , 8 6 6 )}$ & $-0.467^{*}[-0.695,-0.238]$ & & \\
\hline Pregravid & & $2.559^{*}[2.336,2.782]$ & $2.190^{*}[2.039,2.340]$ \\
\hline Pregnancy 1 & & & \\
\hline Interpregnancy & & $2.713^{*}[2.477,2.948]$ & \\
\hline Obese $(\boldsymbol{n}=\mathbf{4 , 3 6 3 )}$ & $-0.683^{*}[-0.777,-0.588]$ & & $1.806^{*}[1.603,2.009]$ \\
\hline Pregravid & & & \\
\hline Pregnancy 1 & & & \\
\hline Interpregnancy & & & \\
\hline
\end{tabular}

Note. Data are presented as average increase or decrease in kilograms [95\% confidence interval] in Pregnancy 1 (GWG), interpregnancy weight loss, and Pregnancy 2 GWG associated with a 5-kg increase in weight during the previous perinatal period (pregravid, Pregnancy 1, and interpregnancy, respectively). Results obtained using a fully recursive simultaneous equations model (full model reported in the Supplemental Appendix in Table A3). Pregnancy 1 GWG, interpregnancy weight loss, and Pregnancy 2 GWG are adjusted for weight changes in prior perinatal weight periods. Data source: Wisconsin births in PeriData.net database, 2006 through 2013. GWG = gestational weight gain.

$* p<.01$.

The effect of weight in one perinatal period on the next was different by BMI category. Normal/underweight women had greater P1 GWG for each 5-kg increase in pregravid weight, whereas overweight and obese women had lower P1 GWG for each 5-kg increment. For each 5-kg increase in P1 GWG, overweight and obese women lost less interpregnancy weight ( $2.5 \mathrm{~kg}$ and $2.7 \mathrm{~kg}$, respectively) than normal-weight women ( $3.2 \mathrm{~kg})$. For each $5 \mathrm{~kg}$ of additional interpregnancy weight loss, overweight women had the greatest subsequent increase in P2 GWG (2.19 kg) followed by obese $(1.81 \mathrm{~kg})$ and normal/underweight women $(1.73 \mathrm{~kg})$.

We displayed the effects of the four perinatal periods on P2 maternal and neonatal outcomes for the full sample (see Figure 2) and for normal weight (see Figure 3), overweight (see Figure 4), and obese (see Figure 5) women as odds ratios (ORs). Odds ratios greater than 1 indicate increased odds of occurrence of the outcome; ORs less than 1 indicate a reduction in the odds of occurrence. Maternal and neonatal outcomes were affected by maternal weight changes during each of the four perinatal periods. Significant independent effects of greater weight change in each period were evident for the following adverse maternal and neonatal outcomes in P2: 
gestational diabetes, gestational hypertension, cesarean birth, LGA, macrosomia, SGA, and low birth weight. No significant effects were identified for the presence of congenital abnormalities and perinatal death.

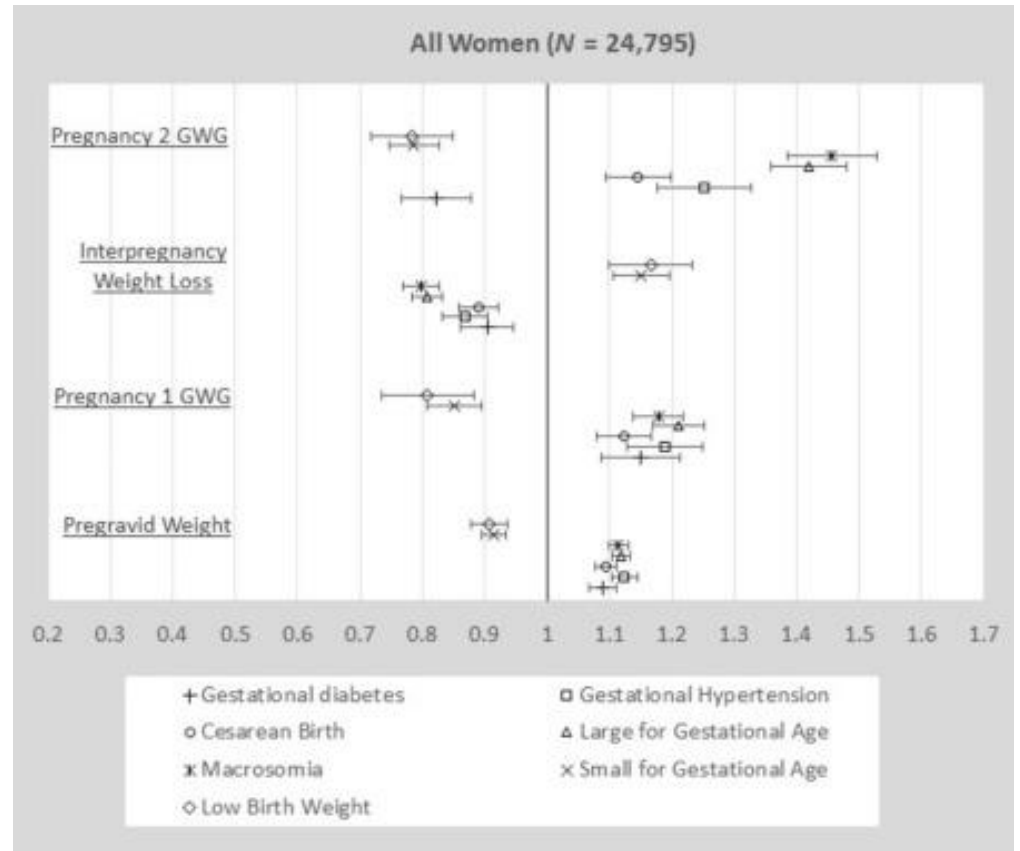

Figure 2. Odds ratios and 95\% confidence intervals for a 5-kg change in weight during a perinatal period on the odds of maternal and neonatal outcomes in the second pregnancy: results for all women. Odds ratio of greater than 1 indicates increased odds of occurrence of the outcome for a 5-kg maternal weight change. Odds ratio of less than 1 indicates decreased odds of occurrence. Calculated with a fully recursive simultaneous equations model. Effects for congenital anomalies and perinatal death were not significant and are not included in this figure. Odds ratios by weight classifications and the full display of results of the simultaneous equation modeling for maternal outcomes and for neonatal outcomes are provided in the Supplemental Appendix in Tables A4, A5, and A6, respectively. Data source: Wisconsin births in PeriData.net database, 2006-2013. GWG = gestational weight gain.

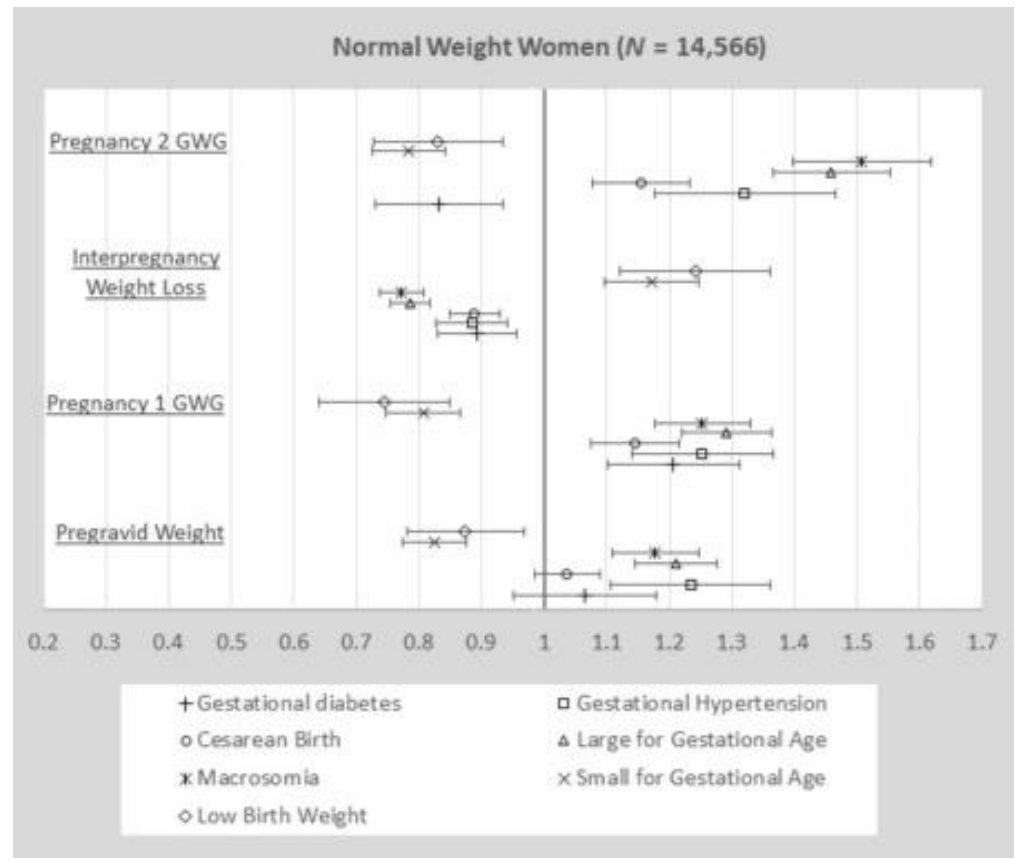


Figure 3. Odds ratios and 95\% confidence intervals for a 5-kg change in weight during a perinatal period on the odds of maternal and neonatal outcomes in the second pregnancy: results for normal-weight (including underweight) women. Odds ratio of greater than 1 indicates increased odds of occurrence of the outcome for a $5-\mathrm{kg}$ maternal weight change. Odds ratio of less than 1 indicates decreased odds of occurrence. Calculated with a fully recursive simultaneous equations model. Effects for congenital anomalies and perinatal death were not significant and are not included in this figure. Odds ratios by weight classifications and the full display of results of the simultaneous equation modeling for maternal outcomes and for neonatal outcomes are provided in the Supplemental Appendix in Tables A4, A5, and A6, respectively. Data source: Wisconsin births in PeriData.net database, 2006-2013. GWG = gestational weight gain.

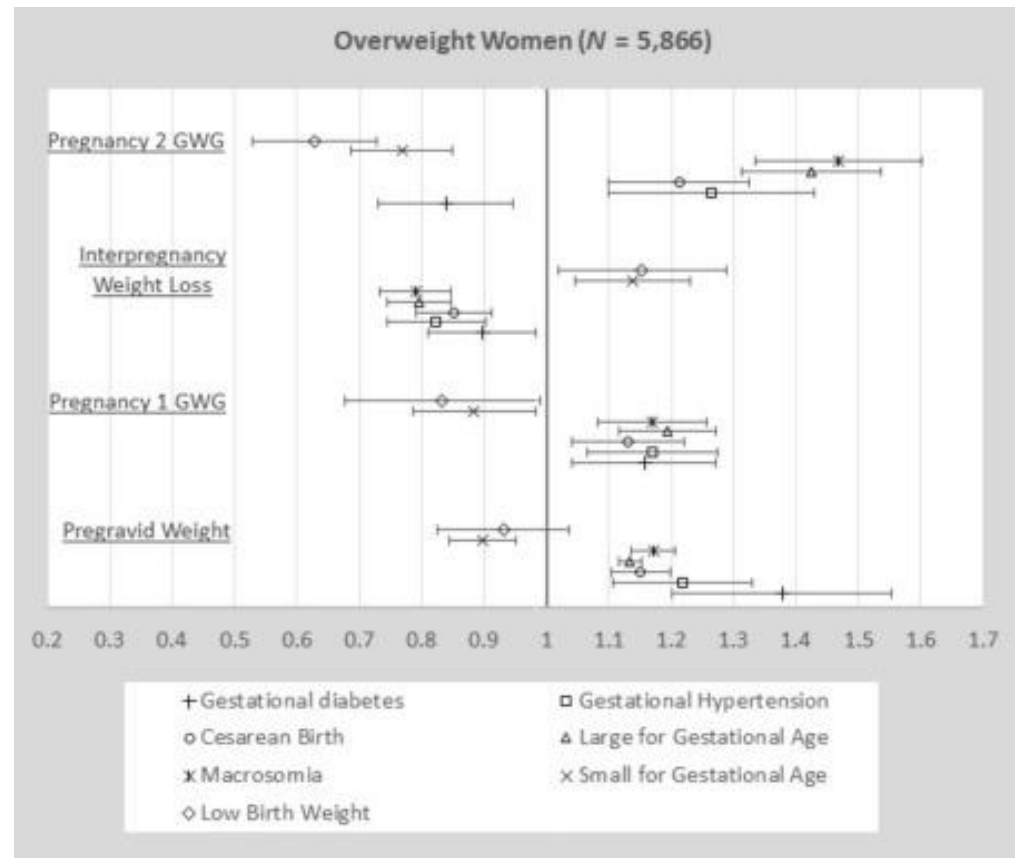

Figure 4. Odds ratios and 95\% confidence intervals for a 5-kg change in weight during a perinatal period on the odds of maternal and neonatal outcomes in the second pregnancy: results for overweight women. Odds ratio of greater than 1 indicates increased odds of occurrence of the outcome for a 5-kg maternal weight change. Odds ratio of less than 1 indicates decreased odds of occurrence. Calculated with a fully recursive simultaneous equations model. Effects for congenital anomalies and perinatal death were not significant and are not included in this figure. Odds ratios by weight classifications and the full display of results of the simultaneous equation modeling for maternal outcomes and for neonatal outcomes are provided in the Supplemental Appendix in Tables A4, A5, and A6, respectively. Data source: Wisconsin births in PeriData.net database, 2006-2013. GWG = gestational weight gain. 


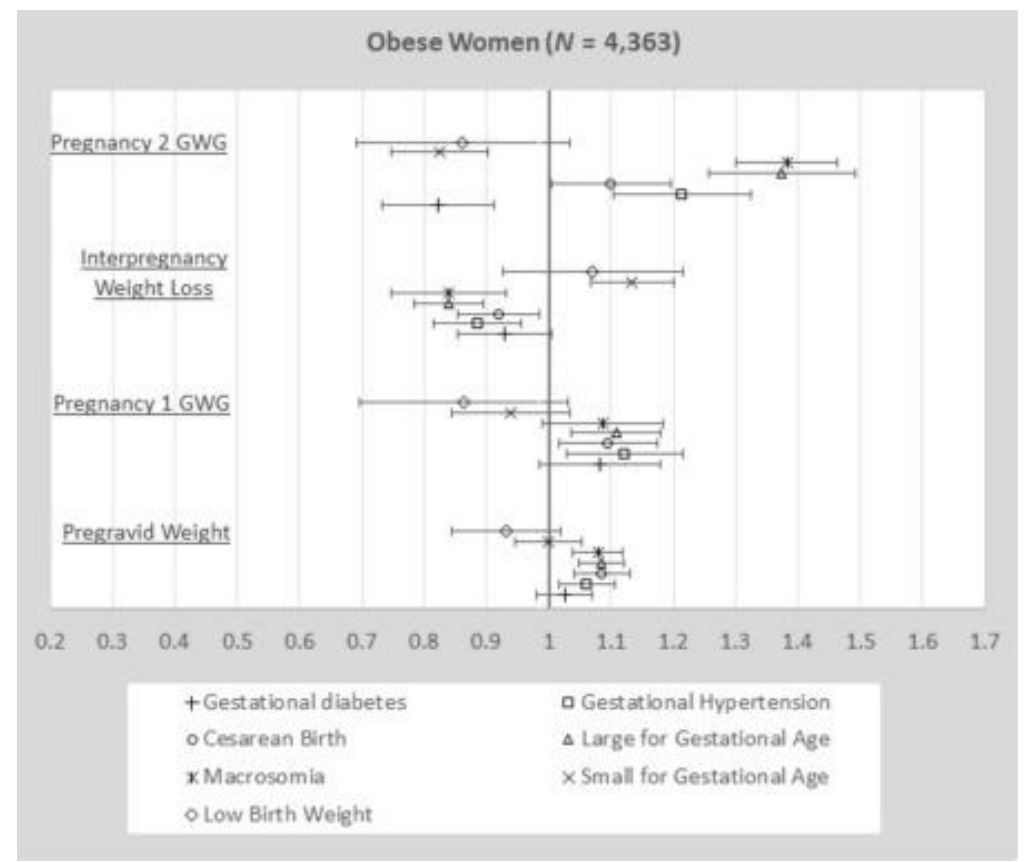

Figure 5. Odds ratios and 95\% confidence intervals for a 5-kg change in weight during a perinatal period on the odds of maternal and neonatal outcomes in the second pregnancy: results for obese women. Odds ratio of greater than 1 indicates increased odds of occurrence of the outcome for a 5-kg maternal weight change. Odds ratio of less than 1 indicates decreased odds of occurrence. Calculated with a fully recursive simultaneous equations model. Effects for congenital anomalies and perinatal death were not significant and are not included in this figure. Odds ratios by weight classifications and the full display of results of the simultaneous equation modeling for maternal outcomes and for neonatal outcomes are provided in the Supplemental Appendix in Tables A4, A5, and A6, respectively. Data source: Wisconsin births in PeriData.net database, 2006-2013.

Maternal and neonatal outcomes in P2 were independently associated with pregravid weight in P1, conditional on weight gain during both gestations and interpregnancy weight loss, but the effect was smaller than the effects of subsequent GWG and interpregnancy periods. Overall, the odds of P2 adverse maternal outcomes were $9 \%$ to $12 \%$ greater (gestational diabetes: $O R=1.089,95 \%$ confidence interval $[\mathrm{Cl}]=[1.068,1.110]$; gestational hypertension: $\mathrm{OR}=1.124,95 \% \mathrm{Cl}[1.104,1.144]$; cesarean birth: $\mathrm{OR}=1.094,95 \% \mathrm{Cl}[1.078,1.111])$ for every 5-kg increase in pregravid weight; however, these associations varied and were not significant across all of the pregravid BMI groups. Pregravid weight was also associated with the odds of P2 adverse neonatal outcomes; specifically, $5 \mathrm{~kg}$ of excess pregravid weight was associated with 11\% greater risk for macrosomia $(\mathrm{OR}=1.114,95 \% \mathrm{Cl}[1.099,1.130])$ and $12 \%$ greater risk for $\operatorname{LGA}(\mathrm{OR}=1.119,95 \% \mathrm{Cl}[1.105,1.13])$ but reduced the odds by $8 \%$ for SGA $(O R=0.915,95 \% \mathrm{Cl}[0.896,0.934])$ and $9 \%$ for low birth weight $(\mathrm{OR}=0.907,95 \% \mathrm{Cl}$ $[0.879,0.936])$.

Pregnancy $1 \mathrm{GWG}$ and interpregnancy weight loss had opposite and offsetting effects on maternal and neonatal outcomes of P2. For each 5-kg increase in P1 GWG, there was $12 \%$ to $19 \%$ greater risk of gestational diabetes $(\mathrm{OR}=1.150,95 \% \mathrm{Cl}[1.090,1.212])$, gestational hypertension $(\mathrm{OR}=1.188,95 \% \mathrm{Cl}[1.131,1.249])$, and cesarean birth $(\mathrm{OR}=1.124,95 \% \mathrm{Cl}[1.081,1.168])$ and a $18 \%$ to $21 \%$ greater risk of $\mathrm{LGA}(\mathrm{OR}=1.211,95 \% \mathrm{Cl}[1.170$, 1.253]), and macrosomia ( $\mathrm{OR}=1.179,95 \% \mathrm{Cl}[1.134,1.226])$; however, each subsequent interpregnancy weight loss of $5 \mathrm{~kg}$ reduced these risks for maternal outcomes by $10 \%$ to $13 \%$ for gestational diabetes (OR $=0.904$, $95 \% \mathrm{Cl}[0.863,0.946])$, gestational hypertension $(\mathrm{OR}=0.868,95 \% \mathrm{Cl}[0.833,0.904])$, and cesarean birth $(\mathrm{OR}=$ $0.890,95 \% \mathrm{Cl}[0.861,0.921])$ and by $19 \%$ to $20 \%$ for LGA (OR $=0.808,95 \% \mathrm{Cl}[0.784,0.832)$ and macrosomia $(\mathrm{OR}=0.799,95 \% \mathrm{Cl}[0.772,0.828])$. Greater P1 GWG was associated with $15 \%$ to $19 \%$ reduction in the risk of 
SGA $(\mathrm{OR}=0.851,95 \% \mathrm{Cl}[0.809,0.894])$ and low birth weight $(\mathrm{OR}=0.809,95 \% \mathrm{Cl}[0.741,0.882])$ in $\mathrm{P} 2$, with subsequent interpregnancy weight loss increasing these risks by $15 \%$ for SGA (OR $=1.151,95 \% \mathrm{Cl}[1.108,1.195]$ ) and $17 \%$ for low birth weight $(\mathrm{OR}=1.166,95 \% \mathrm{Cl}[1.102,1.233])$.

Pregnancy 2 GWG had the strongest association with adverse maternal and neonatal outcomes in P2: each $5 \mathrm{~kg}$ of additional weight gained increased the risk of gestational hypertension by $25 \%(\mathrm{OR}=1.252,95 \% \mathrm{Cl}[1.182$, $1.327])$, cesarean birth by $15 \%(\mathrm{OR}=1.146,95 \% \mathrm{Cl}[1.096,1.198])$, LGA by $42 \%(\mathrm{OR}=1.419,95 \% \mathrm{Cl}[1.362$, $1.479])$, and macrosomia by $46 \%(\mathrm{OR}=1.457,95 \% \mathrm{Cl}[1.390,1.528])$ and reduced by $21 \%$ to $22 \%$ the risk of SGA $(\mathrm{OR}=0.787,95 \% \mathrm{Cl}[0.749,0.827])$ and low birth weight $(\mathrm{OR}=0.784,95 \% \mathrm{Cl}[0.723,0.850])$. An unexpected result was that greater P2 GWG was associated with $18 \%(\mathrm{OR}=0.823,95 \% \mathrm{Cl}[0.771,0.879])$ lesser risk of gestational diabetes.

In the analyses for pregravid BMI, normal/underweight and overweight women had greater increases in risks for the P2 adverse maternal and neonatal outcomes than obese women. Among obese women, the associations of maternal and neonatal risks in P2 with weight changes in the perinatal weight periods were weaker and included nonsignificant associations with gestational diabetes and low birth weight for P1 GWG, interpregnancy, and P2 GWG.

\section{Discussion}

Weight changes during each perinatal period of risk were associated with weight changes in subsequent periods and contributed to risk of adverse maternal and neonatal outcomes in the second pregnancy. Maternal weight changes during first gestation, interpregnancy, and second gestation are strongly interrelated. Greater P1 GWG was associated with greater interpregnancy weight loss and was followed by greater P2 GWG. The magnitude of these maternal weight fluctuations was independently and jointly associated with risk for adverse P2 maternal and neonatal outcomes. Pregravid weight was a poor predictor for the magnitude of these perinatal weight fluctuations; however, it was an independent risk factor for adverse maternal and neonatal outcomes.

The sequential effect of one perinatal period on the next, with each 5-kg incremental change in maternal weight associated with up to $3 \mathrm{~kg}$ of change in weight in subsequent periods, along with the independent associations of these weight fluctuations with maternal and neonatal outcomes in P2, are clinically significant. Monitoring the entire trajectory of pregnancy and interpregnancy weight changes for women of all weight classifications is an important component of health care for women that should be routine and valued. The goal of clinical weight surveillance and management should be to detect and mediate a rollercoaster-type pattern of weight changes because larger weight fluctuations are associated with greater risk of adverse maternal and neonatal outcomes. Incorporating the entire trajectory of prior gestational and interconception weight changes, along with pregravid $\mathrm{BMI}$, in pregnancy risk assessments can provide an opportunity to more effectively identify women at risk for extreme weight fluctuations and poorer birth outcomes. This is particularly important for normal-weight and overweight women, who have larger up-and-down weight cycling than obese women; therefore, they may have the greatest risk for substantial subsequent weight changes and the associated adverse outcomes.

Our findings provide support for the extension of current recommendations for healthy weight management before, during, and after pregnancy. Although emphasizing guidelines for healthy weight before and during pregnancy, clinicians typically provide greater attention and counseling to women with elevated pregravid BMIs to address the well-known risks for adverse maternal and neonatal outcomes. This practice could explain why obese women in our study gained less weight during P1 and why their interpregnancy weight loss and P2 GWG were less than those of normal-weight and overweight women. However, because of the sensitivity of weight discussions, providers may avoid these discussions with normal-weight women, whom they see as at a lesser risk for weight-related complications (Stotland et al., 2010). Our results show that normal-weight women may be 
more susceptible to adverse maternal and neonatal outcomes than overweight or obese women because of larger weight fluctuations across the perinatal periods of risk. Among normal-weight women, an increased focus on recommended gain and loss of gestational weight, nutrition, and physical activity to prevent excessive weight cycling is a proactive strategy to reduce the risk of poor maternal and neonatal outcomes.

Clinical counseling to promote effective weight management should occur at every prenatal, pregnancy, and interpregnancy health care visit, especially for women with excessive gain in pregnancy or larger-than-expected loss after birth. The finding that the effect of P1 GWG on adverse maternal and neonatal outcomes in a second pregnancy can be nearly fully offset by subsequent weight loss is encouraging. When women are counseled and monitored by professionals, interpregnancy weight loss can correct or compensate for excessive GWG.

However, as our findings illustrate, women who lose more during interpregnancy periods are also very prone to gaining more in subsequent pregnancies and experiencing the associated adverse outcomes. Not regaining the excessive weight during a second gestation is equally important and may require significant effort.

A surprising finding in our study was the negative association of the odds of $\mathrm{P} 2$ gestational diabetes with $\mathrm{P} 2$ GWG (i.e., lower P2 gestational diabetes odds associated with greater P2 GWG), evident in the total sample and each BMI category. Given that increased weight in the prior periods was associated with greater risk of gestational diabetes in P2, and because we controlled for the occurrence of gestational diabetes in P1, a plausible explanation may be that women with gestational diabetes during P2 may be more attentive to GWG. Women diagnosed with glucose intolerance or gestational diabetes in a prior pregnancy are advised early in the pregnancy to be more attuned to healthy eating and control of GWG. The inverse effect of GWG on gestational diabetes may not have been evident in earlier studies in which researchers did not examine the association of gestational diabetes with weight gain in the second pregnancy.

Weight surveillance and counselling in each perinatal period are essential to improve maternal and neonatal outcomes.

\section{Limitations}

Our study had several limitations. The generalizability of the findings of this retrospective study is limited by the source data, which included a portion of the population of a single U.S. state; the frequency of in-state repeated pregnancies and the demographic characteristics of the sample are not representative of the state or national population. Advancements in perinatal care and attention by health care providers and women themselves to weight management during childbearing may result in different findings in future studies with similar methods. The study sample included only first and second pregnancies; the effect on later pregnancies was not evaluated because the sample size became smaller, and estimates of effect were less reliable.

We combined underweight and normal-weight women into a single category for analysis. We did not exclude underweight women because pregravid weights are often biased by self-reported weight and because excluding them did not change the results in sensitivity testing. There may be error in weight measures based on when and how weight was obtained and recorded by providers. The data entered by hospital personnel may include standard administrative record errors. The inclusive dates for the sample from 2006 through 2013 biases the sample toward a short interpregnancy interval (mean interpregnancy interval $=20.5$ months). In sensitivity analyses to determine the effects of short ( $<18$ months) and medium (18-60 months) interpregnancy intervals (Copen, Thoma, \& Kirmeyer, 2015), the magnitudes were similar to the main findings, but effect sizes in the short interpregnancy interval subsample were slightly larger than in the medium interpregnancy interval subsample. No information was available in the data set on breastfeeding duration. The effect of breastfeeding duration on weight trajectory in the second pregnancy is not known and should be considered in future replications of this study and in prospective investigations. 


\section{Conclusions}

Larger weight changes in the pregravid and gestational periods of the first pregnancy, the interpregnancy period, and second gestation increase the risk of adverse maternal and neonatal outcomes in the second pregnancy. Our findings indicate the importance of recommendations from the Institute of Medicine (Rasmussen \& Yaktine, 2009) for maternal weight surveillance and management during each individual perinatal weight period in the childbearing cycle and highlight the need for attention to women in all BMI categories, including women of normal pregravid weight. The history of weight changes during and after a prior pregnancy is clinically useful for early identification of women with weight change patterns that increase risk for adverse outcomes. A comprehensive approach to risk assessment and perinatal weight management at each perinatal and interpregnancy health visit for all women regardless of their weight can improve maternal and neonatal outcomes.

\section{Acknowledgment}

Supported by the National Center for Advancing Translational Sciences, National Institutes of Health, through grant number 8 UL1TR000055. The contents are solely the responsibility of the authors and do not necessarily represent the official views of the National Institutes of Health.

Supplementary Material

Download: Download Acrobat PDF file (553KB)

Supplemental Appendix.

\section{References}

Afifi et al., 2012, A.A. Afifi, S. May, V.A. Clark, Practical multivariate analysis (5th ed.). CRC Press, Boca Raton, $\mathrm{FL}(2012)$

American College of Obstetricians and Gynecologists, 2013, American College of Obstetricians and Gynecologists, Committee opinion number 548: Weight gain in pregnancy. Retrieved from https://www.acog.org/Resources-And-Publications/Committee-Opinions/Committee-on-ObstetricPractice/Weight-Gain-During-Pregnancy (2013)

Bogaerts et al., 2013, A. Bogaerts, B.R. Van den

Bergh, L. Ameye, I. Witters, E. Martens, D. Timmerman, ..., R. Devlieger, Interpregnancy weight change and risk for adverse perinatal outcome. Obstetrics \& Gynecology, 122 (5) (2013), pp. 9991009, 10.1097/AOG.0b013e3182a7f63e

Centers for Disease Control and Prevention. (n.d.), Centers for Disease Control and Prevention. (n.d.), Weight gain during pregnancy. Retrieved from https://www.cdc.gov/reproductivehealth/maternalinfanthealth/pregnancy-weight-gain.htm

Chin et al., 2010, J.R. Chin, K.M. Krause, T. Ostbye, N. Chowdhury, C.A. Lovelady, G.K. Swamy, Gestational weight gain in consecutive pregnancies. American Journal of Obstetrics and Gynecology, 203 (3) (2010), pp. 279.e1-279.e6, 10.1016/j.ajog.2010.06.038

Chu et al., 2007, S.Y. Chu, S.Y. Kim, J. Lau, C.H. Schmid, P.M. Dietz, W.M. Callaghan, K.M. Curtis, Maternal obesity and risk of stillbirth: A meta-analysis. American Journal of Obstetrics and Gynecology, 197 (3) (2007), pp. 223-228, 10.1016/j.ajog.2007.03.027

CityMatCH. (n.d.), CityMatCH. (n.d.), Perinatal periods of risk approach. Retrieved from https://www.citymatch.org/perinatal-periods-of-risk-approach

Clayton and Pett, 2011, M.F. Clayton, M.A. Pett, Modeling relationships in clinical research using path analysis part II: Evaluating the model. Journal for Specialists in Pediatric Nursing, 16 (1) (2011), pp. 75 79, 10.1111/j.1744-6155.2010.00272.x

Clogg et al., 1995, C.C. Clogg, E. Petkova, A. Haritou, Statistical methods for comparing regression coefficients between models. American Journal of Sociology, 11 (1995), pp. 1261-1312, 10.1086/230638 
Cnattingius and Villamor, 2016, S. Cnattingius, E. Villamor, Weight change between successive pregnancies and risks of stillbirth and infant mortality: A nationwide cohort study. Lancet, 387 (10018) (2016), pp. 558$565,10.1016 /$ S0140-6736(15)00990-3

Collins et al., 2018, K. Collins, R. Oehmen, S. Mehta, Effect of obesity on neonatal hypoglycaemia in mothers with gestational diabetes: A comparative study. Australian and New Zealand Journal of Obstetrics and Gynaecology, 58 (3) (2018), pp. 291-297, 10.1111/ajo.12717

Copen et al., 2015, C.E. Copen, M.E. Thoma, S. Kirmeyer, Interpregnancy intervals in the United States: Data from the birth certificate and the National Survey of Family Growth. National Vital Statistics Reports, 64 (3) (2015), pp. 1-10

Correa and Marcinkevage, 2013, A. Correa, J. Marcinkevage, Prepregnancy obesity and the risk of birth defects: An update. Nutrition Reviews, 71 (S1) (2013), pp. S68-S77, 10.1111/nure.12058

Endres et al., 2015,

L.K. Endres, H. Straub, C. McKinney, B. Plunkett, C.S. Minkovitz, C.D. Schetter, ..., M.U. Shalowitz, Postpartum weight retention risk factors and relationship to obesity at 1 year. Obstetrics \& Gynecology, 125 (1) (2015), pp. 144-152, 10.1097/AOG.0000000000000565

Gunderson, 2009, E.P. Gunderson, Childbearing and obesity in women: Weight before, during, and after pregnancy. Obstetrics and Gynecology Clinics of North America, 36 (2) (2009), pp. 317332, 10.1016/j.ogc.2009.04.001

Hosmer et al., 2013, D.W. Hosmer Jr., S.A. Lemeshow, R.X. Sturdivant, Applied logistic regression (3rd ed.). Wiley, Hoboken, NJ (2013)

Johansson et al., 2014, S. Johansson, E. Villamor, M. Altman, A.K. Bonamy, F. Granath, S. Cnattingius, Maternal overweight and obesity in early pregnancy and risk of infant mortality: A population based cohort study in Sweden. BMJ, 349 (2014), p. g6572, 10.1136/bmj.g6572

Lindsey and Sheather, 2010, C. Lindsey, S.J. Sheather, Variable selection in linear regression. Stata Journal, 10 (2010), pp. 650-669, 10.1177/1536867X1101000407

Martin et al., 2014, J.E. Martin, A.J. Hure, L. Macdonald-Wicks, R. Smith, C.E. Collins, Predictors of post-partum weight retention in a prospective longitudinal study. Maternal \& Child Nutrition, 10 (4) (2014), pp. 496509, 10.1111/j.1740-8709.2012.00437.x

McDowell et al., 2019, M. McDowell, M.A. Cain, J. Brumley, Excessive gestational weight gain. Journal of Midwifery \& Women's Health, 64 (1) (2019), pp. 46-54, 10.1111/jmwh.12927

Monte et al., 2011, S. Monte, O. Valenti, E. Giorgio, E. Renda, E. Hyseni, M. Faraci, ..., F.F. Di Prima, Maternal weight gain during pregnancy and neonatal birth weight: A review of the literature. Journal of Prenatal Medicine, 5 (2) (2011), pp. 27-30

National Heart, Lung, and Blood Institute Obesity Education Initiative Expert Panel on the Identification, Evaluation, and Treatment of Obesity in Adults, 1998, National Heart, Lung, and Blood Institute Obesity Education Initiative Expert Panel on the Identification, Evaluation, and Treatment of Obesity in Adults, Clinical guidelines on the identification, evaluation, and treatment of overweight and obesity in adults. Retrieved from https://www.ncbi.nlm.nih.gov/books/NBK2003 (1998)

Nehring et al., 2011, I. Nehring, S. Schmoll, A. Beyerlein, H. Hauner, R. von Kries, Gestational weight gain and long-term postpartum weight retention: A meta-analysis. American Journal of Clinical Nutrition, 94 (5) (2011), pp. 1225-1231, 10.3945/ajcn.111.015289

Rasmussen and Yaktine, 2009, K.M. Rasmussen, A.L. Yaktine (Eds.), Weight gain during pregnancy: Reexamining the guidelines, National Academies Press, Washington, DC (2009), 10.17226/12584. Retrieved from

https://www.ncbi.nlm.nih.gov/books/NBK32813

Rowlands et al., 2010, I. Rowlands, N. Graves, S. de Jersey, H.D. McIntyre, L. Callaway, Obesity in pregnancy: Outcomes and economics. Seminars in Fetal and Neonatal Medicine, 15 (2) (2010), pp. 9499, 10.1016/j.siny.2009.09.003

Siega-Riz et al., 2009, A.M. Siega-

Riz, M. Viswanathan, M.K. Moos, A. Deierlein, S. Mumford, J. Knaack, ..., K.N. Lohr, A systematic review of outcomes of maternal weight gain according to the Institute of Medicine recommendations: 
Birthweight, fetal growth, and postpartum weight retention. American Journal of Obstetrics and Gynecology, 201 (4) (2009), pp. 339.e1-339.e14, 10.1016/j.ajog.2009.07.002

StataCorp, 2015, StataCorp, Stata Statistical Software: Release 14 [Computer software]. StataCorp LP, College Station, TX (2015)

Stothard et al., 2009, K.J. Stothard, P.W. Tennant, R. Bell, J. Rankin, Maternal overweight and obesity and the risk of congenital anomalies: A systematic review and meta-analysis. JAMA, 301 (6) (2009), pp. 636650, 10.1001/jama.2009.113

Stotland et al., 2010, N.E. Stotland, P. Gilbert, A. Bogetz, C.C. Harper, B. Abrams, B. Gerbert, Preventing excessive weight gain in pregnancy: How do prenatal care providers approach counseling? Journal of Women's Health, 19 (4) (2010), pp. 807-814, 10.1089/jwh.2009.1462

Stubert et al., 2018, J. Stubert, F. Reister, S. Hartmann, W. Janni, The risks associated with obesity in pregnancy. Deutsches Arzteblatt International, 115 (16) (2018), pp. 276-283, 10.3238/arztebl.2018.0276

Tabet et al., 2015, M. Tabet, L.H. Flick, M.G. Tuuli, G.A. Macones, J.J. Chang, Prepregnancy body mass index in a first uncomplicated pregnancy and outcomes of a second pregnancy. American Journal of Obstetrics and Gynecology, 213 (4) (2015), pp. 548.e1-548.e7, 10.1016/j.ajog.2015.06.031

Talge et al., 2014, N.M. Talge, L.M. Mudd, A. Sikorskii, O. Basso, United States birth weight reference corrected for implausible gestational age estimates. Pediatrics, 133 (5) (2014), pp. 844-853, 10.1542/peds.20133285

Villamor and Cnattingius, 2006, E. Villamor, S. Cnattingius, Interpregnancy weight change and risk of adverse pregnancy outcomes: A population-based study. Lancet, 368 (9542) (2006), pp. 11641170, 10.1016/S0140-6736(06)69473-7

Wallace et al., 2016, J.M. Wallace, S. Bhattacharya, D.M. Campbell, G.W. Horgan, Inter-pregnancy weight change and the risk of recurrent pregnancy complications. PLOS ONE, 11 (5) (2016), Article e0154812, 10.1371/journal.pone.0154812

Weesie, 1999, Weesie, J. (1999). Sg121: Seemingly unrelated estimation and the cluster-adjusted sandwich estimator. Stata Technical Bulletin, 52, 34-47. College Station, TX: Stata Press. 